\title{
Planes de Universitas Odontologica para el bienio 2019-2020*
}

Universitas Odontologica Plans for the Biennium 2019-2020

\section{JORGE ENRIQUE DELGADO TRONCOSO}

Pontificia Universidad Javeriana, Bogotá, Colombia. Universidad de Pittsburgh, Pittsburgh, Pennsylvania, Estados Unidos. jed41@ @itt.edu. https://orcid.org/0000-0002-1127-9516

\section{Sandra Cecilia Delgado Troncoso \\ Universitas Odontologica. Medellín, Colombia. sandelga15@gmail.com. https://orcid.org/0000- $\underline{0001-8515-5199}$}

*Editorial.

${ }^{\mathrm{a} C}$ Correspondencia: jed41@ @itt.edu

doi: https://doi.org/10.11144/Javeriana.uo37-79.pduo

Cómo citar: Delgado JE, Delgado SC. Planes de Universitas Odontologica para el bienio 2019 y 2020. Univ Odontol. 2018 jul-dic; 37(79). https://doi.org/10.11144/Javeriana.uo37-79.pduo

\section{Palabras clave}

plan; dossiers temáticos; revisiones integrativas; revisiones sistemáticas; conocimiento útil 


\section{Áreas temáticas}

odontología; revistas científicas

\section{Keywords}

plan; integrative reviews of literature; systematic reviews of literature; thematic dossiers; useful knowledge

\section{Thematic fields}

dentistry; scientific journals

La desmesurada dependencia de las universidades, los académicos y las revistas científicas de Colombia y el mundo de los servicios bibliográficos de indización y resumen nos han generado una gran preocupación por la deformación y mercantilización que han sufrido la comunicación científica y los propósitos mismos de la educación superior y la investigación. Nos hemos preguntado: ¿Qué hacer para que Universitas Odontologica tenga un mayor impacto en la sociedad? La sociedad aquí debemos entenderla como el gremio odontológico, las facultades/escuelas de odontología y la sociedad civil a la que sirven los profesionales y equipos humanos de la salud oral. Con esto en mente, hemos decidido promover y publicar más dossiers temáticos y revisiones de la literatura, bien sean narrativas, integrativas o sistemáticas. Hasta el momento, hemos incluido un dossier en casi cada número de la revista. El próximo dossier será parte del N. ${ }^{\circ} 80$ del primer semestre de 2019 sobre "Políticas públicas de salud bucal en Iberoamérica", que ha resultado de una bonita colaboración con nuestros colegas del Observatorio 
Iberoamericano de Políticas Públicas en Salud Bucal. A comienzos del 2019, lanzaremos los tres siguientes dossiers para ser publicados dos en cada número. Los temas de los dossiers que ya hemos elegido son: "Odontología y discapacidad en Iberoamérica"; "VIH/sida y salud bucal"; y "Educación odontológica".

Nos hace falta el tema del cuarto dossier y los subsiguientes. Para buscar que los dossiers tengan mayor acogida, vamos a preguntar a nuestros seguidores en las redes y en nuestros comités asesores sobre los temas que les gustaría ver cubiertos. Asimismo, vamos a invitar a sociedades de especialistas iberoamericanas, estudiantes de pregrado y posgrado, expertos, docentes e investigadores a escribir sobre los temas de su experticia. Para asegurarnos que las revisiones que recibamos y publiquemos hagan un aporte nuevo al conocimiento, vamos a pedir a los autores que vayan más allá de simplemente relatar lo que posiblemente ya está escrito en otros lugares. Esta es una práctica común que vemos en los libros publicados de la disciplina y las revisiones de tema que nos envían a Universitas Odontologica. El éxito y publicabilidad de las revisiones que recibamos en adelante radicará en el análisis crítico o evaluación de la evidencia disponible, la identificación de los horizontes (esto es, hasta dónde ha llegado el conocimiento y hacia dónde se dirigen los avances) y qué problemas particulares prácticos y de conocimiento fundamental aun están por resolverse. Nosotros hemos enfatizado esto siempre, pero ahora le daremos mayor empuje. Debemos recordar que aun dependemos de obtener citaciones y que nuestro propósito sigue siendo divulgar el conocimiento de punta acerca de y originado en Iberoamerica. 